\title{
WaterWOLF: Water Watch on Load Flow
}

\author{
C. Schantz ${ }^{1}$, J. Donnal ${ }^{1}$, S. Leeb ${ }^{1}$, P. N. Marimuthu ${ }^{2}$ \& S. Habib ${ }^{2}$ \\ ${ }^{I}$ Massachusetts Institute of Technology, USA \\ ${ }^{2}$ Kuwait University, Kuwait
}

\begin{abstract}
We have developed WaterWOLF, a new electronic system and signal processing algorithm for evaluating flow components in pipes. These techniques nonintrusively convert an existing flow meter into a high resolution wireless meter for determining flow rates in real-time. These techniques exploit new tunneling magneto-resistive materials (TMR) for detecting very small magnetic fields generated by certain types of flow meters. The sensors are nonintrusive, requiring no new access to the flow stream. A pipe distribution network, e.g., for water or oil production or potentially even gas utilities, can not only deliver a commodity like water but can also serve as its own sensor for monitoring water flow and the operation of individual water consuming appliances. Continuous measurements of water consumption can be fed back to the user to help find opportunities for conservation. Additionally, high resolution flow sensing can improve leak detection accuracy. This is important for countries with significant water challenges like Kuwait.
\end{abstract}

Keywords: utility monitoring, water consumption, smart water meter, flow rate sensing, non-intrusive sensing, tunnelling magnetoresistive sensors.

\section{Introduction}

Production of potable water may have large financial and energy cost, especially in desalination dependent countries like Kuwait. Reducing demand can lower the energy footprint of water and promote sustainability. Finding water waste requires effective sources of information. A new generation tunneling magneto resistive (TMR) sensors (Ikeda et al. [1]), opens the door to retrofit high resolution flow sensors on existing water meter infrastructure. Many water customers are individually metered with mechanical water meters. These meters 
contain magnets attached to elements that rotate in response to flow. We present a strap on sensor and algorithm to sense flow rate with high resolution.

The drive to digitize customer level water flow is in high gear. Advances in communications technology are being leveraged to track consumption via smart water meters (Oracle [2]). The Kuwait Ministry of Electricity and Water has recently signed a deal to receive 170000 automatic meter reading (AMR) units for use in government buildings and residences (Elster [3]). AMR units collect flow totals similarly to conventional water meters and transmit this information to the utility. This avoids the need for in-home meter reading for bill collection. AMR meter systems report in fixed volume increments of large $(>100 \mathrm{~L})$ size, and transmit only when polled by a central system (Ernesto et al. [4]). Smart water meters improve on AMR capabilities with finer volume increments and time resolution, and streaming information transfer.

Irrespective of the connectivity scheme, the "wet" side of AMR and smart water meters are often identical to conventional mechanical meters. A positive displacement element rotates in response to flow, causing rotation of a magnet (Arregui [5]). In mechanical meters, a follower magnet on the dry side of the meter increments a mechanical register to track total volume. In electronic meters, a reed switch or similar device generates a series of pulses for accumulation. The pulse based volume quantization of virtually every AMR or smart meter to date is a fundamental limitation that has implications in flow trace accuracy and water balance leak detection thresholds.

This limitation is eliminated by the high sensitivity of our TMR sensor and the Instantaneous Frequency (IF) signal processing algorithm developed below. The high performance of the sensor permits external attachment. The signal IF is related to flow rate through the meter's volume per rotation constant. The increased information available from our scheme opens up new avenues in household water consumption tracking and research. Furthermore, detailed consumption feedback has been proven an effective non-monetary tool for encouraging latent conservation behaviour in consumers across a number of utilities like electricity and gas (Faruqui et al. [6]).

\subsection{Similar work}

External sensors to convert the magnetic drives of water meters into pulses for tracking flow are not new. Home automation hobbyists have presented circuits and sensors to track their own water meters (Cheung [7]). Commercial strap on sensors and data logger hardware like the MM100EL Flow Recorder are also available (F. S. Brainard and Company [8]). Additionally, some modern mechanical meters are smart enabled (Hauber-Davidson and Idris [9]), and contain pulse probe insertion ports. Larger (non residential) capacity meters may even feature standardized pulse output terminals. However, all these schemes use pulse based volume quantization. Researchers have also investigated new integrated circuit architectures for magneto resistive sensors to reduce hysteretic effects in smart metering/magnet rotation tracking applications (Zhang et al. [10]). Our circuit uses an external coil compensation scheme to mitigate hysteresis in commercially available TMR sensors. 


\section{Water challenges and metering in Kuwait}

Kuwait is an arid country and its natural water resources are below the $1000 \mathrm{~m}^{3} /$ year/capita scarce line defined by World Health Organization (Falkenmark and Widstrand [11]). Kuwait's water supply depends mainly on non-conventional water resources such as desalination plants. Fresh water consumption in Kuwait has increased with population, and presently, the per capita water consumption is augmented to 500 liters/day [12]. The average water footprint of Kuwait is reported as $2072 \mathrm{~m}^{3} /$ year/capita, which is $50 \%$ above the global average water footprint figure $1385 \mathrm{~m}^{3} /$ year/capita [13].

The daily statistics report obtained from Ministry of Water and Electricity in 2013 has shown an average increase of $7 \%$ in domestic water consumption from 2012 levels [14]. This is due to two factors. The first factor is the residential houses are large in size, and almost all houses are multi-storied with a minimum of two to three floors. The second factor is that the Kuwait Government heavily subsidizes the cost of water, which is considered to be one of the reasons for increased water consumption. Retrofit of purely mechanical water meters with our strap-on sensor to can provide the benefits of direct consumption feedback without the cost of meter replacement. This information stream will also improve the efficiency of bill collection, an important aspect to promote responsible water use.

Kuwait also has distribution leaks. One sign of leakage in water distribution networks is increased urban groundwater levels (Lerner [15]). Data from Kuwait Institute for Scientific Research Water Resources Center show urban ground water levels rising over the period from 1988 to 2004 in the cities of Hawally (population: 164,000) and Kuwait City (population: 2.3 million). Khaitan, a suburb of Kuwait City, has seen a rise of approximately 5 meters in this time period (Akber [16]). One method to detect leaks in distribution networks is to establish District Metering Areas (DMA) and perform a water balance between inflow and outflow in the DMA. Even if time referenced measurements from all meters bounding the DMA are available, pulse based volume accumulation readings will always have an undershoot error between zero and one volume increment. Since these errors are cumulative, i.e. they all point in the same direction, summation of meter readings compounds the error, increasing the minimum detectable leak size. Low cost non-pulse based digitization of existing customer water meters can enable more sensitive water balance leak detection in existing distribution systems.

\subsection{Residential water meters}

In Kuwait, positive displacement (PD) water meters are used to measure the total consumption of water to many individual houses. PD type meters are insensitive to flow rate dependent accuracy problems of other meter options and are therefore popular for metering water customers due to variable flow rate demands and financial accuracy concerns. The water meter in figure 1(a) is an oscillating piston style PD meter, which records the water flow in one direction 


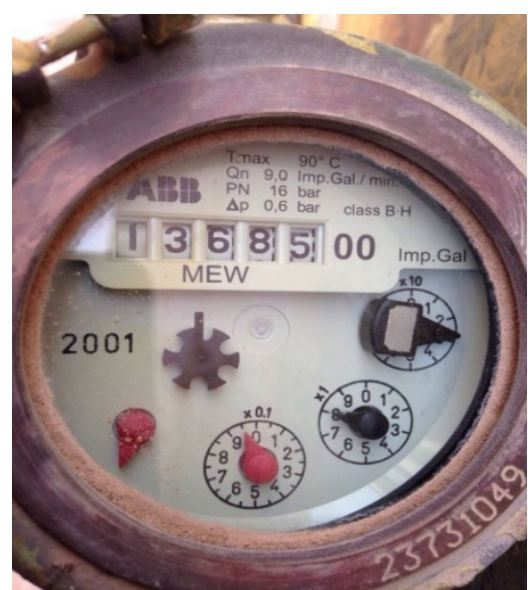

(a)

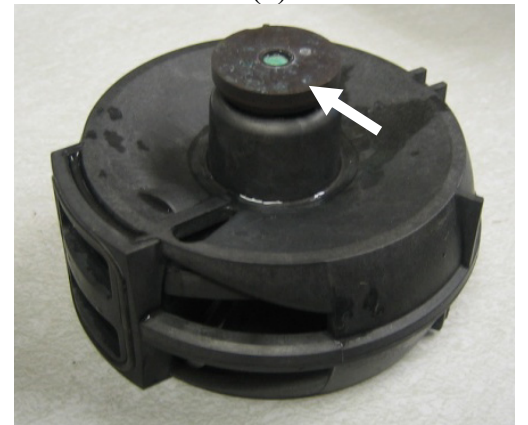

(c)

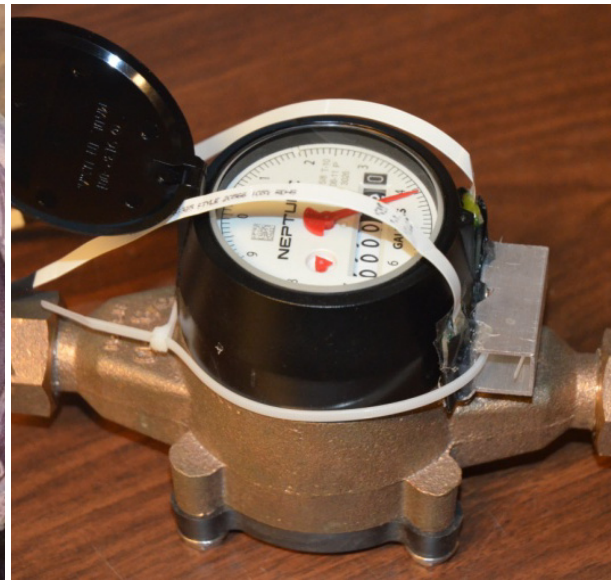

(b)

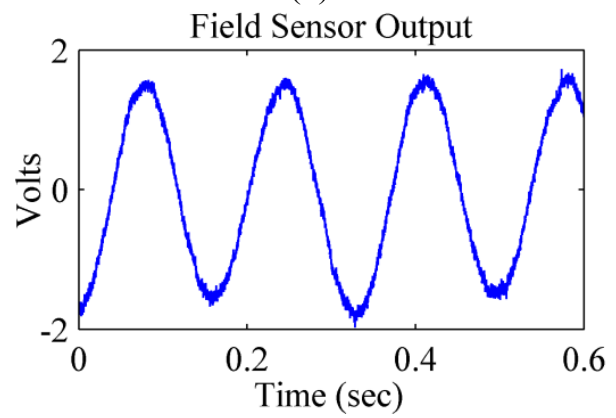

(b)

Figure 1: (a) PD water meter from the Ministry of Electricity and Water (MEW) at a Kuwaiti residence. (b) Side view of similar meter with attached sensors. (c) PD meter measurement cartridge with top magnetic disk at arrow and (d) Plot of magnetic waveform distortion near meter.

only by means of volumetric measuring method. The meter is equipped with a piston placed within a measuring chamber, which gets rotated by the water flow. Each piston revolution is equivalent to a known volume of water. The piston movement is transferred by reduction gearing and a magnetic drive to a straight reading in imperial gallons [17]. The meter in figure 1(b) is a nutating disk PD meter, shown with two sensors attached. Flow causes a disk shaped plate to wobble in, sweeping out a fixed volume per cycle in its chamber. The wobbling motion is converted to rotation via a linkage attached to magnetic drive.

Mechanical meters are common in Kuwait. A standard design is 2 inches in diameter, and is constructed to withstand pressures as high as 16 bar and temperature of $90^{\circ} \mathrm{C}$ to suit Kuwait's outdoor weather during summer. The popular class B meter has a nominal flow rate of 9.0 imperial gallons/min for 
residential service. All flanged meters of $40 \mathrm{~mm}$ nominal diameters are known as class B type meters.

\subsection{Meter magnetics}

The PD mechanism and wet side magnetic element of a nutating disk water meter are shown in figure 1(c). The magnet element is a disk containing two north poles and two south poles in an alternating configuration. This two pole pair disk is designed to couple rotational motion to a set of follower magnets in the register head. The quality and symmetry of the magnetic field produced by this coupled set of magnets is not tightly controlled. A measurement of the field strength near the disk during rotation shows a sinusoid with harmonic distortion. Figure 1(d) shows the variation in field strength from one pole to the next. This variability from pole to pole, and likely from meter to meter, has implications for instantaneous frequency extraction, described in the next section.

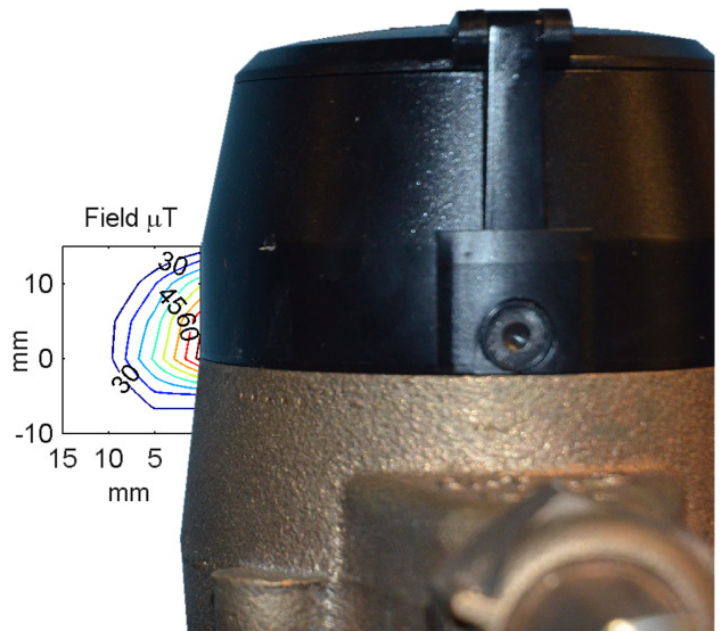

Figure 2: Peak to peak variation in meter's external field.

Sensor positioning will also affect the measured signal. Higher order components of the field, e.g. any quadrupole component, will decay faster with distance than the dipole component. As an external attachment, the sensor position will be at least a couple diameters from the magnetic disk. At this range the dipolar component of each magnetic pole pair will dominate. Our sensors are sensitive on one magnetic axis which is oriented vertically for a horizontally mounted meter. A map of the peak to peak variation in field strength in a radial plane measured during meter rotation is shown in figure 2. The map is an interpolation of a $5 \mathrm{~mm}$ square grid of vertical axis field measurements of an Aim Instruments I-prober 520. The field map shows the region of highest field strength corresponds to the area level with the gap between the wet magnetic disk and the register's follower magnets. 


\subsection{Meter signal model}

The magnetic field at a fixed point on the periphery of the water meter, given in eqn (1), is a function of the rotation angle $\theta$ of the magnets inside the meter. The $c_{2}$ coefficient will dominate the other coefficients for a two pole pair disk.

$$
H(\theta)=\sum_{n=-\infty}^{\infty} c_{n} e^{i 2 \pi n \theta}
$$

Assuming an accurate meter with no internal leaks, $\theta$ will depend on the total volume of fluid that has passed through the meter. Total volume since an arbitrary starting point is the integral of the flow rate over the same period, relating $\theta(t)$ to flow rate $f(t)$ through eqn (2). The volume per rotation factor $V_{\text {rot }}$ is a mechanical parameter of the meter design.

$$
\theta(t)=\frac{2 \pi}{V_{\text {rot }}} \int_{0}^{t} f(\tau) d \tau
$$

The flow rate $f(t)$ is clearly related to the derivative of the phase of the harmonic components of the magnetic field $H(t)$. For a mono-component analytic signal the IF is the instantaneous phase derivative, and many algorithms for estimating IF from analytic signals may be employed to deduce instantaneous flow rate. Water meter magnetic fields are nearly mono-component due to the dominance of the $c_{2}$ coefficient. Hilbert transform based methods to convert real valued field measurements into complex analytic signals are inaccurate for signals with low frequency content (Boashash [18]). Because flow does not always occur, the signal in question may have extended periods of dc. We therefore require two sensors mounted with circumferential separation to produce a second real valued signal. We also require an angular correction scheme to combine the two real valued signals into a suitable analytic signal for IF estimation. Using two sensors also allow flow direction determination.

\section{Electronics}

Sensing high quality waveforms in the weak magnetic field in the proximity of a domestic water meter requires new sensor technology. We have developed a prototype compensated magnetic sensor circuit built around a TMR element.

\subsection{Sensor selection}

Many types of magnetic sensor exist. Lenz and Alan [19] offer a good overview. We desire a small form factor without complicated diving and support electronics, ruling out fluxgate technologies. Cost concerns immediately rule out SQUID based devices. We desire a sensor that is not speed dependent and ruling out coil based sensors. High sensitivity will relax placement tolerance making the installation easier. While the peak field measured in outside the meter are equivalent to the earth's magnetic field at approx $60 \mathrm{uT}$, placement $1 \mathrm{~cm}$ from 
the peak location will result in significantly reduced field. Therefore, we specify that an acceptable sensor would be capable of sensing an order $10 \mathrm{uT}$ peak to peak field change with high quality. Current hall effect sensors do not meet this specification. The magneto resistive type TMR sensor offers the combination of small form factor, DC capability, simplicity, bipolar response, and sensitivity required for our application.

The TMR effect describes a mechanism for resistance change in material due to applied magnetic fields. The mechanism was first understood in the $1970 \mathrm{~s}$ but had little practical value due to relatively small changes in material resistance (Julliere [20]). Recent advancements using new materials and fabrication techniques have improved the sensitivity of TMR devices. Modern state of the art sensors show up to $600 \%$ change in relative resistance at room temperature [1]. Interest in these devices has increased as they have become integrated into high density magnetic disk drives and MRAM (Hoberman [21]).

The STJ-340 is a TMR Wheatstone bridge sensor produced by MircoMagnetics. The sensor has four active TMR elements, arranged in a Wheatsone bridge architecture (Micro Magnetics [22]). Changes in the field induce an imbalance in the bridge which can be measured by a differential amplifier. While the STJ-340 can detect very small fields $(25 \mathrm{mV} / \mathrm{G}$ as constructed), there are two significant challenges in using it as an accurate magnetic waveform sensor. First DC offset errors quickly saturate the sensor output. The offset errors from the environment and from imbalance in the bridge itself (which can be up to $10 \%$ ) must be removed before applying any significant gain to the output. More troubling though is the sensor's nonlinear response to large changes in the applied field. Even with proper amplification and DC offset removal, step changes in the field produce non-linear responses in the sensor output.

\subsection{Sensor circuit}

The sensor and circuit shown in figure 3(a) and 3(b) addresses both the DC offset and the non-linearity problems of the TMR sensor. The DC offset error is corrected by an integrator connected to the REF pin of the instrumentation

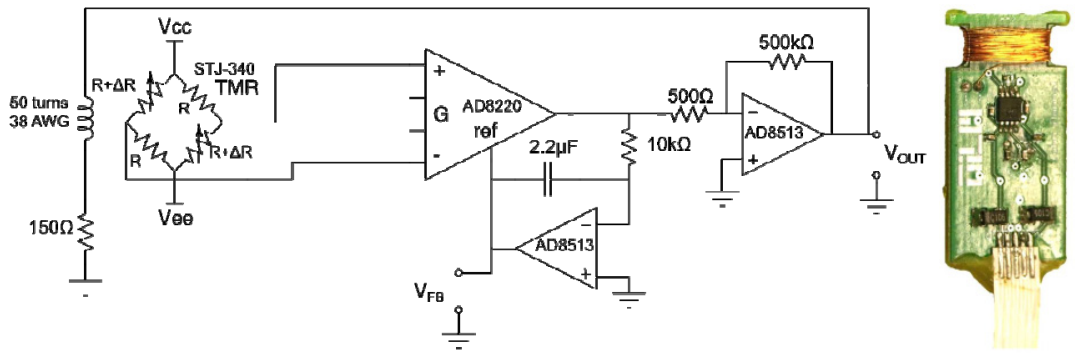

(a)

(b)

Figure 3: (a) Schematic of sensor circuit. (b) photo of sensor. 
amplifier. Any DC component is subtracted off the amplifier output resulting in a purely AC signal. The REF pin is sampled to recover pseudo DC information, as discussed in the algorithms section. This output is then fed through a high gain stage which drives an air core solenoid wrapped around the STJ-340. The current through this solenoid builds a magnetic field that opposes the applied field, creating a feedback loop that zeros the operating point of the STJ-340. Keeping the sensor element exposed to very small fields improves the sensor linearity and increases its range of operation. The current driven in the compensation solenoid is sensed as a voltage across a 150 resistor. The final stage provides additional gain.

\section{Signal processing for IF estimation}

Each sensor gives two outputs, the AC coupled magnetic field denoted $\mathrm{H}(t)$ and the low frequency/DC containing reference pin voltage of the instrumentation amplifier, denoted $\mathrm{R}(t)$. Two sensors are required to track flow direction and must be combined in quadrature to form an analytic signal for IF estimation. If mounting considerations or unknown magnetic pole counts in the meter result in non quadrature sensor placement i.e. the sensors are not ninety degrees separated in the magnetic space angle, then a constant angular correction must be performed. The magnetic space angle is equal to the physical space angle multiplied by the pole pair count of the magnet disk. The correction angle $\phi_{M}$ in eqn (3) may be found by maximizing the positive valued frequency content and minimizing the negative valued frequency content of $z(t)$ using a segment of sample data taken during system install.

$$
z(t)=H_{1}(t)-e^{i * \phi_{M}} H_{2}(t)
$$

Then a block processing algorithm is used to return pseudo DC content to $z(t)$. Finally IF is calculated through a two step process designed to significantly attenuate the extraneous harmonics of $z(t)$ arising from non symmetric construction of the meter's magnetic elements.

\subsection{Pseudo DC response recovery}

Our implementation automatically corrects imbalance in the TMR bridge, which are influenced by exposure to DC fields. The correction is done by shifting the reference voltage against which the instrumentation amplifier reads the sensor. A side effect of this correction is distortion of turn-on flow transients. Before flow starts, the magnetic elements in the flow meter are stationary and the sensor is exposed to a constant DC field value. The offset corrector will adjust the sensor output in response, causing the output of the I-amp to trend to zero in the absence of flow. When flow resumes the integrator will modify its reference correction based on the average of new alternating field, which is generally different from the stationary field preceding flow. This distorts the sensor output during the initial few seconds of flow. These distortions are unwanted and can potentially cause difficulty with IF estimation algorithms. Turn-on transient 


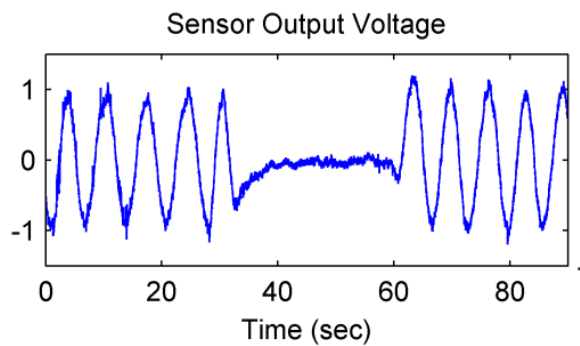

(a)

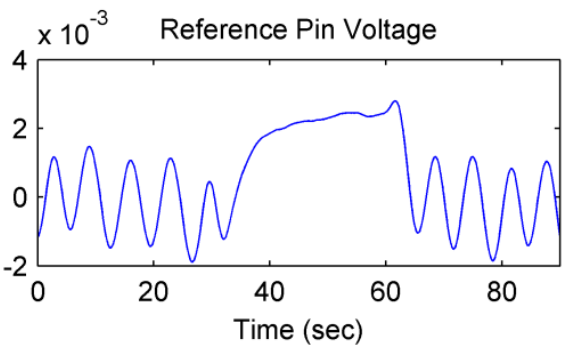

(b)

Figure 4: (a) plot of sensor output channel, showing slow flow, a pause, and flow resuming. (b) Corresponding $R_{1}$ channel, filtered.

distortions can be seen in figure 4(a). Figure 4(b) shows a low pass filtered $R_{1}$ channel containing the reference voltage offset corrections.

Because stationary periods expose the sensor to a random DC field value, the offset correction can be modeled as piecewise time varying. The procedure to recover DC content by reintegrating $R_{1}$ with $H_{1}$ must also be done in a piecewise manner. The first step is to determine the boundaries between regions of flow. This is done by high pass filtering the analytic signal constructed in eqn (3), and comparing its absolute value to a threshold. Signal regions with amplitude envelopes below the threshold are considered no flow regions. This operation sets a minimum flow rate, which is governed by the time scale of the integrator and the high pass filter's pole locations. For a desired minimum flow rate detection level, one may design the threshold, filter, and integrator pole accordingly.

$$
H(t)-(S * R(t)+O) \equiv H_{c}(t)
$$

For every segment, the linear scaling $S$ and offset $O$ scalars to minimize eqn (4) are found via least squares. Then $H_{c}(t)$ is calculated. This operation corrects the distortion of flow segments, but sets the stationary segment values to zero. The no flow segment means are corrected with a simple additive shift using the final value of the preceding flow segment as an approximation to the true DC value of the no flow segment. The segments are then re-assembled to give the pseudo DC corrected signal shown in figure 5(a). Any discontinuities created by this algorithm are inconsequential for the IF estimation algorithm employed next.

\subsection{Instantaneous frequency extraction}

The concept of instantaneous frequency is applicable only to mono-component signals (Boashash [18]). The magnetic signals recorded for flow rate determination consists of a majority component proportional to the spin rate of the mechanical flow metering device, but harmonics and noise are also present. Because the frequency of interest can vary, flow rate estimation is improved by employing a variable center frequency band pass filter to precisely track and pass the dominant component. This filter is implemented via a heterodyning process. 
First, an initial IF estimate is used to create a mixing signal which is multiplied by the analytic signal to shift the frequency range of interest to DC for low pass filtering. The results are then multiplied by the inverse of the mixing signal to return the signal to the original domain, allowing an improved IF estimate.

For discrete analytic signals, IF can be estimated from the derivative of the instantaneous phase. The inherent numerical derivative makes signal noise a primary concern. We use the Kay-Tretter algorithm described in Kay [23]. It is based on an assumption of short time linear phase variation and operates on relative phase differences between points to avoid wrapping problems. A discontinuity will only contribute one erroneous differential phase value to the estimation window. The linear slope of the phase in the estimation window is computed in a least squares sense. The window length sets the frequency response vs. noise susceptibility tradeoff. The initial IF estimate for the mixing signal used to filter out harmonics is performed with a longer window than the final IF estimate. Figure 5(b) contains the result of the frequency tracking filter.

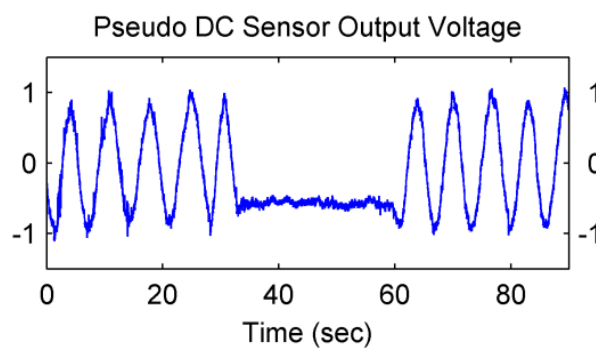

(a)
Tracking Filtered Sensor Output

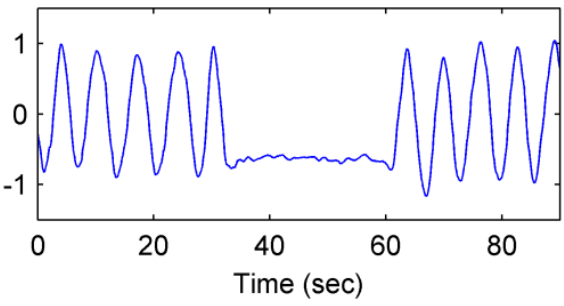

(b)

Figure 5: (a) $H_{c}(t)$ showing pseudo DC correction. (b) Filtered signal.

\section{Field test}

WaterWOLF was tested at a New England residence. The sensors were attached to the home's water meter using a zip-tie similar to figure 1(b). Sensor placement was near the border between the brass meter body and the plastic register head. Acceptable signal amplitude was confirmed with an oscilloscope. The meter's volume per rotation coefficient was found via least square fit of a series of flow steps from the home's basement sink. During this test all other loads in the home were turned off. The sink flow was measured with a commercial electronic meter designed for attachment to garden hose taps. Flow rate step transitions were abrupt to give clear indication in the data.

During the field test household water fixtures were sequentially activated and deactivated. One objective was to determine if automatically operated water loads gave a distinct inrush flow rate signature. A processed annotated flow trace example is shown in figure 6 . The data reveals that inrush transients are not consistent even for automatically operated fixtures like a toilet reservoir refill valve. The cause is the difference in initial flow friction resisting the turn-on 
flow. Sudden flow increase from a quiescent pipe state will initially have lower resistance to flow before turbulence develops. If turbulence already exists in the pipes, however, the increased friction will act immediately.

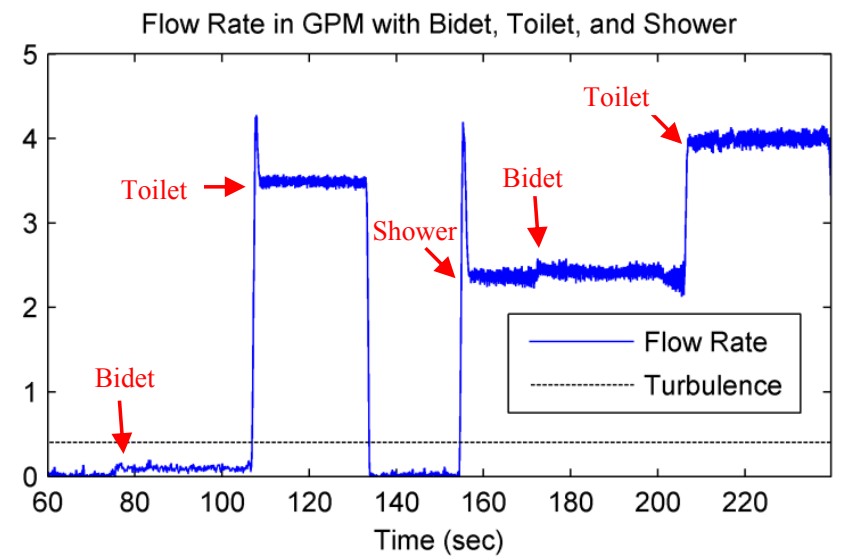

Figure 6: Field test flow trace example.

\section{Conclusion}

In this paper, we develop a nonintrusive flow monitor for water, oil, and gas pipeline networks. We have shown field test results carried out at a New England residence to analyse the flow rate signatures of household water loads. We plan to conduct similar experiments in Kuwaiti residences.

\section{Acknowledgement}

The authors would like to acknowledge the support from Kuwait-MIT Center for Natural Resources and the Environment research grant no. 2013-5505-02.

\section{References}

[1] Ikeda, S., et al. "Tunnel magnetoresistance of $604 \%$ at $300 \mathrm{~K}$ by suppression of $\mathrm{Ta}$ diffusion in $\mathrm{CoFeB} / \mathrm{MgO} / \mathrm{CoFeB}$ pseudo-spin-valves annealed at high temperature." Applied Physics Letters 93(8), 2008.

[2] Smart Metering for Water Utilities, Oracle, online www.oracle.com/us/industries/utilities/046596.pdf

[3] Elster press release www.elster.com/en/press-releases/2010/1444595

[4] Ernesto, A.-P., et al. "Modeling Automatic Meter Reading Water Demands as Nonhomogeneous Point Processes." Journal of Water Resources Planning and Management 140(1), pp. 55-64, 2012. 
[5] Arregui, F., Enrique C., Ricardo C., (eds). Integrated Water Meter Management. IWA Publishing, 2006.

[6] Faruqui, A., Sergici, S., Sharif, A. The impact of informational feedback on energy consumption-A survey of the experimental evidence, Energy, 35(4), pp. 1598-1608, 2010.

[7] Municipal water meter monitor, E. Cheung, online www.edcheung.com/automa/water.htm

[8] Meter Master 100EL Brochure, F.S. Brainard and Company online www.meter-master.com/products/flow recorders/mm100el af.html

[9] Hauber-Davidson, G., Idris, E. Smart water metering Water 33(3), pp. 3841, 2006.

[10] Zhang, Z., et al. An Ultra Low-Power MR* 1) Sensor for a Smart Water Meter or a Smart Gas Meter. Key Engineering Materials 543 pp. 418-421, 2013.

[11] Falkenmark, M, and Widstrand, C., Population and Water Resources: A Delicate Balance, Population Bulletin, 47(3), pp. 1-36, 1992.

[12] Statistical Report on Water Pipeline Network 2007-2013; Statistics Department and Information Center, Kuwait.

[13] Water Footprint, www.waterfootprint.org

[14] Daily Statistics Report on Fresh Water Consumption; Statistical Department and Information Center, Ministry of Electricity and Water, on July 31, 2013, Kuwait.

[15] Lerner, D. N., Groundwater recharge in urban areas, Atmospheric Environment. Part B. Urban Atmosphere 24(1) pp. 29-33, 1990.

[16] A. Akber, Water Security in Kuwait: Aspirations and Realities, Kuwait Institute for Scientific Research Water Resources Center.

[17] Water Meter Manual, http://www.abb.com

[18] Boashash, B. Estimating and interpreting the instantaneous frequency of a signal. I. Fundamentals. Proceedings of the IEEE 80(4) pp. 520-538, 1992.

[19] Lenz, J. and Alan S. E., Magnetic sensors and their applications, Sensors Journal, IEEE 6(3) pp. 631-649, 2006.

[20] Julliere, M., Tunneling between ferromagnetic films, Physical Review Letters A, 54(3) pp. 225-226, 1975.

[21] Hoberman, B, The emergence of practical MRAM, Crocus Technologies Online. www.crocustechnology.com/pdf/BH\%20GSA\%2OArticle.pdf.

[22] STJ-340: Four Element Bridge Magnetic Sensor Data Sheet, Micro Magnetics.

[23] Kay, S. A fast and accurate single frequency estimator, Acoustics, Speech and Signal Processing, IEEE Transactions on 37(12) pp. 1987-1990, 1989. 